\title{
Apps para el aprendizaje de idiomas en la Universidad de Murcia
}

\section{Apps for foreign language learning at the University of Murcia}

\author{
$\mathrm{M}^{\mathrm{a}}$ Encarnación Carrillo-García \\ Universidad de Murcia, Murcia, España \\ mariaencarnacion.carrillo@um.es \\ Antonia Cascales-Martínez \\ Universidad de Murcia, Murcia, España \\ antonia.cascales@um.es \\ Amando López Valero \\ Universidad de Murcia, Murcia, España \\ amandolo@um.es
}

\begin{abstract}
Resumen
El artículo presenta los resultados de una investigación cuyo propósito fue conocer el uso que se realiza en el ámbito universitario de los dispositivos móviles y de las aplicaciones utilizadas en las materias impartidas de lenguas extranjeras. A partir de un diseño de investigación ex post facto descriptivo, siendo la muestra participante 86 alumnos de la Facultad de Educación de la Universidad de Murcia. Se utilizó un cuestionario para recoger datos confeccionado ad hoc, validado mediante el procedimiento de validación por juicios de expertos y la fiabilidad fue analizada mediante el alfa de Cronbach. Los resultados evidencian que las Apps son herramientas adecuadas para el aprendizaje de los idiomas si bien no se utilizan como tales en los diferentes entornos educativos, universitarios y no universitarios.
\end{abstract}

Palabras clave: educación tecnológica, aprendizaje de lenguas, enseñanza universitaria, idioma extranjero, dispositivos móviles.

\begin{abstract}
The article presents the results of a research whose purpose was to know the use made in the university field of mobile devices and the applications used in the subjects taught in foreign languages. Based on an ex post facto descriptive research design, 86 students from the Faculty of Education of the University of Murcia participated in the sample. A questionnaire was used to collect data made ad hoc, validated by the validation procedure by expert judgments and the reliability was analyzed using Cronbach's alpha. The results show that Apps are adequate tools for language learning, although they are not used as such in the different educational, university and non-university environments.

Keywords: technological education, language learning, university education, foreign language, mobile devices.
\end{abstract}

\section{Introducción}

Las aulas universitarias están llenas de nativos digitales (Fajardo, Villalta, Salmerón, 2016) es decir, jóvenes que desde su nacimiento se han visto rodeados por variedad de dispositivos tecnológicos, ordenadores portátiles, tablets, iPads, móviles, etc... que han estado a su alcance, los han utilizado y se puede decir que en mayor o menor medida son competentes en su uso. Esta revolución tecnológica ha llegado a las aulas, bien de la mano de los alumnos, de los propios docentes o como reclamo de la sociedad, y por tanto no podemos obviarla; al contrario, desde el ámbito universitario debemos hacerla 
nuestra aliada para mejorar el proceso de enseñanza-aprendizaje en su conjunto, y no verse relegada a un aumento de recursos educativos. Burbat (2016) pone de relieve esta situación en el contexto de la enseñanza-aprendizaje de las lenguas extranjeras.

Ozuorcun y Tabak (2012) definen el Mobile Learning como un modelo de educativo que permite a los estudiantes obtener materiales de aprendizaje en cualquier lugar y en cualquier momento usando tecnologías móviles e Internet (véase figura 1). Adquiriendo pleno sentido al respecto, las Apps como recursos de enseñanza-aprendizaje dado el creciente número que hay en el mercado. Entendemos por Apps, una aplicación diseñada para dispositivos móviles, que permite realizar uno o varios trabajos de forma simultánea. Las Apps las podemos encontrar libres o de pago y las podemos descargar directamente en nuestros dispositivos. Es cierto que han alcanzado un auge impresionante, pero también es cierto que las Apps suelen usarse en periodos cortos de tiempo (Henze et al. 2011).

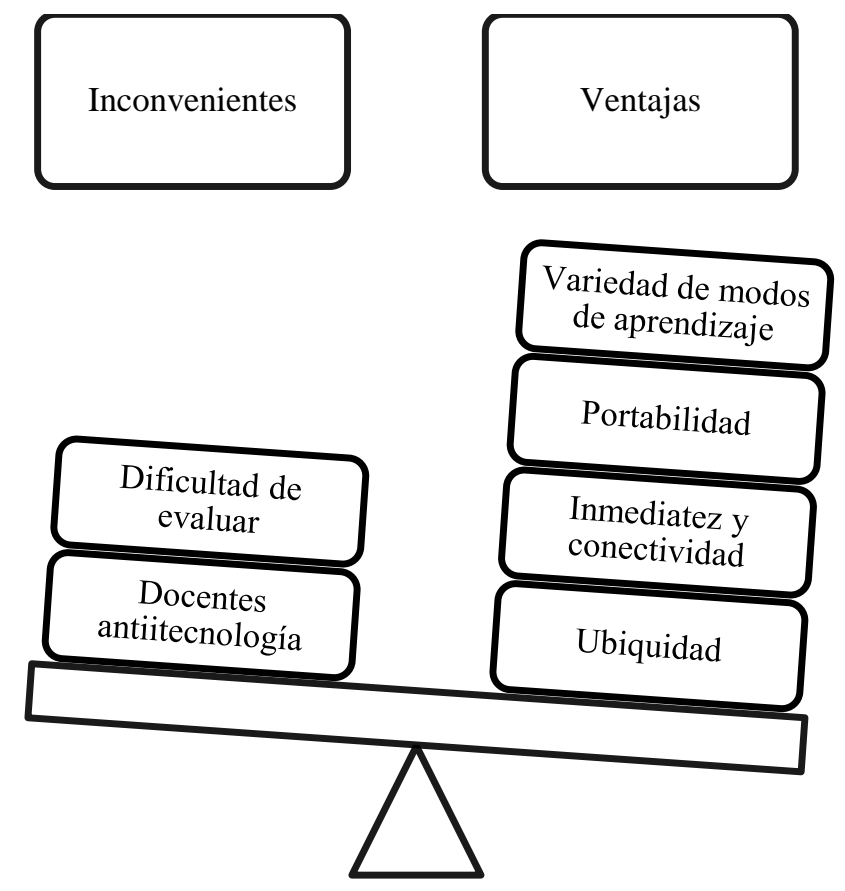

Figura 1: Ventajas e inconvenientes del Mobile Learning

A los procesos de enseñanza y aprendizaje de las lenguas extranjeras cuyo fin último es el desarrollo de la competencia comunicativa (Bumfit y Johnson, 1991; Brown, 1994) en la lengua objeto de estudio, se suma ahora la necesidad de desarrollar diferentes competencias relacionadas con el uso de las tecnologías en el alumnado, como la competencia digital y la competencia informacional (Área, 2010).

La alfabetización digital del docente, implica por parte del docente un impacto positivo por la integración de las tecnologías en el aula, conllevando 1 adquisición de diferentes competencias y un aprendizaje (Del Moral, Villalustre y Neira, 2013). Destacando un proceso de formación permanente para los docentes, debido al continuo desarrollo y evolución vertiginosa de la tecnología, así como la inclusión en el proceso de enseñanza-aprendizaje, ya sea de manera formal o informal (Avello, López y Vázquez, 2016). El hecho de que el docente incorpore tecnologías en su docencia, señala un planteamiento de cambio hacia nuevos retos y desafíos, lo que implica conocimiento,

Apps para el aprendizaje de idiomas en la Universidad de Murcia. $\mathrm{M}^{\mathrm{a}}$ Encarnación CarrilloGarcía, Antonia Cascales-Martínez, y Amando López Valero. 
habilidades, cambio de actitudes y tiempo; este cambio supone, además de una inversión de tiempo, dado que es un proceso planificado y ajustado a la materia, también está relacionado con el modelo educativo, el cual involucra los procesos de enseñanza-aprendizaje, la institución, los estudiantes y los docentes (Zempoalteca Durán, B., Barragán López, J., González Martínez, J. \& Guzmán Flores, T., 2017). En ese sentido Correa, Fernández, Gutiérrez, Losada y Ochoa-Aizpurua (2015) indican que la alfabetización digital debe mantener el aprendizaje docente y ofrecer este conocimiento al alumnado para que puedan narrarse mediática, audiovisual y colaborativamente por medio de la incorporación de las redes sociales, los videos y las herramientas de trabajo colaborativo.

La alfabetización digital del alumnado tendrá como consecuencia el desarrollo de su competencia digital la cual conlleva ser competente en lenguajes, tecnología, procesos de producción y difusión, procesos de recepción e interacción, ideología y valores, y dimensión estética; pero afectaría directamente a dos de ellas: la dimensión linguística y la tecnológica. Para Hernández (2015) los ambientes virtuales de aprendizaje se entienden como espacios digitales diseñados para lograr objetivos de aprendizaje; y pueden ser a nivel individual o colectivo, en forma sincrónica o asíncrona y promover la aprensión de significados. En este sentido lo digital se refiere a la convergencia de las tecnologías de computación y de comunicación para generar medios que estén disponibles en Internet, la Web o en cualquier plataforma que los incluya. López y Aguaded (2015) señalan que fomentar la alfabetización mediática es misión de la comunidad educadora, que debe asumir la responsabilidad de demandar propuestas de formación del profesorado que incluyan materias docentes en las que se eduque al respecto.

La literatura en este campo (Marcelo, Yot y Mayor, 2015) explica que los resultados de investigaciones previas ponen en relieve que en las aulas universitarias no se han integrado de manera exitosa una amplia gama de tecnologías para apoyar el proceso de enseñanza-aprendizaje. Y defienden que para que se produzca una integración exitosa de las tecnologías en los procesos de enseñanza-aprendizaje el profesorado debe centrar su atención no tanto en los recursos tecnológicos, sino en las experiencias de aprendizaje que diseñan y para las que las tecnologías resultan adecuadas. Cascales, Gomariz y Martínez-Segura (2016) señalan la relevancia de que los docentes realicen un trabajo de preparación de recursos y materiales tecnológicos, al tiempo que diseñan la secuencia que se debe seguir en los aprendizajes. Öngün y Demirag (2015) apuntan a que la relación entre las tareas que realizan el alumnado y las ayudas de la tecnología ha sido ignorada, de manera que se ha obviado la amplia gama de facilidades que los avances tecnológicos proporcionan para que el alumnado consiga realizar con éxito la realización de sus tareas. Área, Hernández y Sosa (2016) inciden en que las investigaciones futuras acerca de los modelos de integración didáctica de las tecnologías en el aula deben centrarse en qué medida el profesorado se readapta a la llegada de los dispositivos móviles en muchos casos aportados por el propio alumnado. Y Piñeiro y Costa (2016), en su estudio sobre los juegos de realidad alternativa como recurso educativo en el ámbito universitario, explican que la docencia universitaria debe adaptarse al contexto tecnológico-social en el que viven sus protagonistas; y que el aula, como espacio educativo y de aprendizaje, no debe ser ajena a lo que sucede fuera de ella.

Apps para el aprendizaje de idiomas en la Universidad de Murcia. $\mathrm{M}^{\mathrm{a}}$ Encarnación CarrilloGarcía, Antonia Cascales-Martínez, y Amando López Valero.

Página 3 de 18 
En este contexto es necesario hacer referencia a la competencia digital, entendida como aquella competencia relacionada tanto con el profesorado como con el alumnado, de manera que se convierte en una competencia que adquiere una doble dimensión, pues afecta a los dos agentes involucrados en los procesos de enseñanza y aprendizaje. En relación a la primera, la competencia digital docente, Padilla-Hernández, GámizSánchez y Romero-López (2018) explican que es aquella competencia digital que debe tener el profesorado universitario, e inciden en que la práctica y el desarrollo de dicha competencia ejerce una gran influencia en su propia evolución profesional. A este respecto, Prendes, Espinosa y Sánchez (2018) en su investigación sobre la situación actual de las universidades en España y su dotación tecnológica en relación a la situación del profesorado universitario, destacan que la competencia profesional docente está estrechamente relacionada con la competencia digital, de manera que el profesorado que adquiera una mayor competencia digital, también repercutirá en una mayor profesionalización de su docencia. Investigaciones como las de LázaroCantabrana, Gisbert-Cervera, Silva-Quiroz (2018) inciden en la necesidad de centrar el tema de estudio de la competencia digital del profesorado universitario mediante un proceso de evaluación que defina el concepto competencia digital, por lo que proponen una evaluación basada en un sistema de rúbrica en el que se midan las dimensiones que la definen en relación a una "Dimensión Didáctica, curricular y metodológica", a una "Dimensión de planificación, organización, gestión de espacios recursos tecnológicos digitales", otra "Dimensión relacional, ética y de seguridad" y finalmente una "Dimensión personal y profesional". Como vemos existe una necesidad de evaluación de la competencia digital docente, pues de esta evaluación podrá plantearse una formación adecuada según las necesidades que se plantee en, y como fruto de esto se obtendrá una mayor profesionalización del docente universitario, y así lo apuntan también Tejada y Pozos (2018), pues según las conclusiones de su investigación, se hace necesario un modelo de formación del docente universitario que integre la competencia digital en el perfil profesional del mismo.

En relación a la competencia digital del alumnado, entendemos que se hace necesaria una formación en el ámbito digital del alumnado independientemente de la materia de estudio que realice, ya que como afirman Monroy y Hurtado (2018) es una competencia transversal, cuyo conocimiento es muy necesario tanto para desarrollar la labor profesional como para su vida personal, debido a que las tecnologías digitales se encuentran inmersas en nuestra sociedad y forman parte de nuestro día a día. Como vemos el desarrollo de la competencia profesional del profesorado universitario y del alumnado, depende también, y en esto existe coincidencia en ambos agentes del proceso educativo, en que se desarrolle de manera adecuada la competencia digital de ambos, pues capacita a los profesionales de cualquier ámbito para el desarrollo de su trabajo. Y para ello estudios como el de Cabero, Arancibia, Valdivia y Aranedas (2018) reclaman la necesidad de establecer planes de formación para la adquisición de la competencia digital del alumnado en relación a la evaluación de la información obtenida en el espacio digital, así como la necesidad de dotar de una mayor capacitación de los docentes para incorporar estos recursos en sus aulas.

Por ello, es necesario abordar el tema de la alfabetización digital del alumnado, debido a que se convertirá desde las perspectiva de nuestro estudio en uno de los principales objetivos a desarrollar en las aulas de educación superior; en esta dirección Ojeda, Reyes y Robles (2018) previa evaluación de las necesidades de formación del

Apps para el aprendizaje de idiomas en la Universidad de Murcia. $\mathrm{M}^{\mathrm{a}}$ Encarnación CarrilloGarcía, Antonia Cascales-Martínez, y Amando López Valero. 
alumnado, abogan por el diseño y el establecimiento de programas de alfabetización digital para así poder constituir ambientes educativos que enriquezcan los saberes docentes en el uso de herramientas digitales. La importancia de la alfabetización del alumnado en tecnología es de suma importancia, tal y como apuntan González, GómezMillan y Rubio (2017) pues aquellos que se encuentren en dificultades en el acceso o manejo de las nuevas tecnologías de la información y la comunicación, serán consideradas personas que se encuentren situadas en la brecha digital, y como consecuencia se encuentran en claro riesgo de exclusión social. Rodriguez, Méndez y Martín (2018) explican que a pesar de que España es uno de los mayores consumidores de contenidos digitales de todo el mundo, la competencia multimedia e informacional de su ciudadanía es todavía insuficiente, e incluso inciden en que hay un vacío formativo al respecto en el ámbito universitario, por lo que advierten de la necesidad de una correcta alfabetización informacional y digital en los estudiantes universitarios.

En lo que se refiere a los procesos de enseñanza-aprendizaje de las segundas lenguas y las lenguas extranjeras, Izquierdo (2008) entiende que éxito depende de un buen modelo pedagógico, debe apoyarse en los principios de aprendizaje activo, colaborativo y autónomo, opciones variadas de interactividad, comunicación sincrónica y asincrónica, actividades o tareas relevantes y creativas y evaluación y retroalimentación continuos. Las tecnologías son herramientas que pueden emplearse para exponer a los estudiantes a una segunda lengua o lengua extranjera en la medida que existe una amplia gama de actividades de comprensión y producción (Izquierdo, Cruz, Aquino Zúñiga, Sandoval Caraveo \& García Martínez, 2017). Blake (2015) explica que el Computer-Assisted Language Learning puede contribuir al desarrollo de las cuatro destrezas del alumnado: escuchar, hablar, leer y escribir; y varias investigaciones corroboran que el uso de dispositivos móviles ayuda a mejorar la competencia comunicativa de las segundas lenguas, como nos explican Rico y Agudo (2016) en la conclusión a su investigación acerca del aprendizaje móvil del inglés a través de videojuegos; en concreto, destacan que la tecnología móvil facilita un aprendizaje interactivo, ubicuo e individualizado; y tras su análisis concluyen que se puede constatar la idoneidad de utilizar material didáctico mediante dispositivos móviles, en su caso a través de video juegos, para el aprendizaje de las diferentes competencias comunicativas y la formación cultural implícita. Demouy, Jones, Kna, Jukulska-Hulme y Eardley (2016) explican también, en su investigación sobre cómo utilizar los dispositivos móviles para la enseñanza de las lenguas, que entre los recursos que se pueden utilizar se encuentran las websites, Apps, Youtube, Google Map, redes sociales o se pueden utilizar para realizar tareas fuera del espacio de la clase. En el mismo orden de cosas, Macaro y sus colegas (2011), señalan que el empleo de las tecnologías para el aprendizaje de una segunda lengua puede favorecer procesos cognitivos, psicológicos y socio-afectivos en los estudiantes. Dado que los estudiantes perciben la tecnología como un recurso excelente para difundir los conocimientos adquiridos en el aula (Felix, 2004) y les motiva fomentando actitudes positivas (Durán-Fernández \& Barrio-Barrio, 2007).

Por otro lado, nos encontramos con estudios como el de Alvarado, Cohelo y Dougherty (2016) en la que nos presentan y analizan tres aplicaciones útiles para la enseñanza de las lenguas, en concreto: Kahoot, Edpuzzle, Audioboom; tres aplicaciones que trabajan varias destrezas lingüísticas y que ayudan a desarrollar la competencia comunicativa del alumnado, concluyendo los autores que promueven el aprendizaje basado en tareas, ayudan a repasar de una manera práctica, dinámica y divertida la lengua objeto de

Apps para el aprendizaje de idiomas en la Universidad de Murcia. $\mathrm{M}^{\mathrm{a}}$ Encarnación CarrilloGarcía, Antonia Cascales-Martínez, y Amando López Valero.

Página 5 de 18 
estudio, fomentando además la autonomía de cada estudiante en la medida que les ayuda a responsabilizarse de su propio aprendizaje. En esta línea, Gunter, Campbell, Braga, Racilan y Souza (2016) analizan también varias aplicaciones que se pueden utilizar en el aula de lenguas, así como su capacidad de promover el aprendizaje de forma lúdica, en el listado de aplicaciones analizadas se encuentran: Word Search, Duolingo: Lear nLanguages Free, Scramble: The Free Word Searh, 4 Pics 1 Word, Aworded (Apalabrados), Word Search, Hanging With Friends, Word Search, 4 Pics 1 Word Puzzle Plus, Learn English 6,000 Words, LearnLanguages-busuu, What'sthe Phrase Free, Word Search, Word Search Puzzles, Babbel-Learn Languages, 4 Pics 1 Song, New Words With Friends, Word Crack Free; en concreto, revisan el número de descargas, el número de usuarios, y ordenan las aplicaciones por categorías: Puzle, Educación, y Vocabulario; y concluyen que las aplicaciones Bussu y Duolingo, se caracterizan por favorecer el aprendizaje a través del juego y además el aprendizaje que promueven puede ser utilizado en contextos reales de uso de las lenguas. Lindaman y Nolan (2015) indican que las Apps ayudan a trabajar de manera práctica los contenidos de la lengua e incrementan exponencialmente las oportunidades de aprendizaje.

Moreno, Leiva y Matas (2016) relacionan el Mobile Learning con otros términos como Gamificación (aprendizaje basado en juegos) y Realidad Aumentada (visualización directa o indirecta de elementos del mundo real o aumentados con elementos virtuales), analizando en su artículo varias aplicaciones y programas para su uso en el ámbito de la enseñanza y aprendizaje de idiomas desde un enfoque comunicativo; entre los que nos encontramos aplicaciones ya revisadas por otros autores como Duolingo o Busuu, y otras como Wlingua, Vocabal, Lingualia, Funland, Juega y aprende idiomas, SpanishLeaningGames, Bitstrips, Qiver, Chromville, Zookazam, Layar, Aurasma, Augment; y acompañan su estudio de varias propuestas didácticas con ellas; y defienden que es necesario la concienciación y la correcta formación del profesorado en la selección y creación de aplicaciones informáticas acordes con las necesidades y características de su alumnado, pues además de tener conocimientos técnicos de los recursos educativos en los que se convierten las aplicaciones es necesario que dicha formación incluya contenidos pedagógico-didácticos.

Otras investigaciones analizan el desarrollo de las diferentes competencias a través de los dispositivos móviles, así Martínez, Olivencia y Terrón (2016) estudian el desarrollo de la competencia escrita en la enseñanza de lenguas extranjeras a través del uso de dispositivos móviles; y en concreto, se analiza la viabilidad del uso del servicio de mensajería instantánea WhatsApp Messenger para establecer un intercambio escrito entre alumnos de Francés Lengua Extranjera de nivel intermedio, confirmándose la viabilidad de la propuesta didáctica y destacando que después de un periodo de doce semanas utilizando este tipo de mensajería en lengua extranjera el alumnado presenta mejoras significativas en las destrezas escritas en lengua francesa. Destacamos de esta propuesta las diferentes posibilidades que nos brindarían, en el área de lenguas, las diferentes formas de comunicación que nos ofrecen las redes sociales: Facebook, Twitter, Google+, etc; en cuanto a la lengua escrita, así como aquellas otras aplicaciones que implican el uso de la lengua oral como por ejemplo Skipe, la videoconferencia en Facebook, etc.

Y en el caso de la enseñanza-aprendizaje de la lengua oral, otros estudios analizan el papel de las aplicaciones que trabajan los aspectos orales de la lengua como es el caso de García, Mosquera, y Marro (2016) en su trabajo sobre recursos innovadores en la

Apps para el aprendizaje de idiomas en la Universidad de Murcia. $\mathrm{M}^{\mathrm{a}}$ Encarnación CarrilloGarcía, Antonia Cascales-Martínez, y Amando López Valero.

Página 6 de 18 
enseñanza de la lengua inglesa en la etapa de educación infantil, entre los que destacamos los relatos digitales (Digital Storytelling) que definen como una técnica de narración digital que consiste en la recreación de material audiovisual a partir del uso y edición de imágenes, animaciones o música. En concreto analizan las aplicaciones: Myths and Legends, una página web donde pueden encontrarse gran cantidad de relatos narrados en inglés adecuados a la etapa de educación infantil (E2BN Websites, 2006); Someries, que es un portal que ofrece historias relatadas por niños y otras inventadas por ellos, con la posibilidad de que aparezcan subtítulos (EducationalTechnology and Mobile Learning, 2012); y Domo Animate, una aplicación que permite la creación de relatos digitales basados en el cómic, con la posibilidad de introducir personajes, música, o efectos especiales (GoAnimateforSchools, 2016). Y finalmente decir, al respecto del mobil learning, que autores como Sánchez, Olmos y García-Peñalvo (2017) concluyen en su investigación la necesidad de incluir programas específicos de formación en mobil learning, de cara a la mejora progresiva de la aceptación de estos dispositivos por parte de los alumnos a medida que avanza su proceso educativo.

Con el objeto de desarrollar nuestro estudio al respecto, en la investigación que presentamos nos hemos centrado en conocer la percepción que tienen los alumnos universitarios de los Grados de Educación Infantil y Primaria y del Máster de Formación del Profesorado en el uso de Apps como recursos educativos en los contextos de enseñanza y aprendizaje de las lenguas extranjeras en el ámbito universitario. Siendo conscientes del uso creciente de los dispositivos móviles en nuestra sociedad, pretendemos recabar información acerca de las diferentes aplicaciones que se pueden utilizar con ellos y que ayudan a trabajar las destrezas orales, las destrezas escritas, los contenidos gramaticales, de vocabulario, socioculturales y fonéticos para la enseñanza de las lenguas extrajeras; cómo se usan y en qué medida, así como el conocimiento y la apreciación que el alumnado tiene de los mismos; en concreto, entre los estudiantes de los grados de Educación Primaria y Educación Infantil y el alumnado que cursa los estudios de Máster en Formación del Profesorado de la Facultad de Educación de la Universidad de Murcia (España).

\section{Metodología}

En este trabajo tratamos de conocer la percepción que tienen los alumnos universitarios sobre el uso de las Apps para el aprendizaje de los idiomas en el ámbito universitario; con el fin de conocer el grado de conocimiento, en qué medida conocen sus características, qué habilidades desarrollan en el aprendizaje de idiomas y en el contexto universitario y, finalmente, cómo influyen en el aprendizaje a lo largo de toda la vida. De este objetivo se derivan los siguientes objetivos específicos:

1. Analizar la percepción que tiene los alumnos sobre las Apps para el aprendizaje de los idiomas.

2. Analizar las posibles diferencias entre la percepción que tienen los alumnos sobre las Apps para el aprendizaje de los idiomas en relación al género y a la titulación.

3. Determinar la relación existente entre las diferentes habilidades (comprensión oral y escrita y expresión oral y escrita) para aprender un idioma con Apps.

Apps para el aprendizaje de idiomas en la Universidad de Murcia. $\mathrm{M}^{\mathrm{a}}$ Encarnación CarrilloGarcía, Antonia Cascales-Martínez, y Amando López Valero. 


\section{Diseño y muestra}

El diseño de este estudio se caracteriza por ser un estudio ex post facto descriptivocuantitativo; concretamente presentamos un estudio que adopta un planteamiento descriptivo con diseño de grupo único, cuya finalidad exploratoria trata de medir la percepción que tiene los alumnos universitarios del Grado de Maestro, Educación Infantil y Educación Primaria; y Máster del Formación del Profesorado de la Universidad de Murcia de las Apps para el aprendizaje de Idiomas.

Los participantes en esta investigación han sido 86 alumnos de la Facultad de Educación de la Universidad de Murcia. La selección ha respondido a un muestreo intencional respetando el procedimiento de grupo intacto. En cuanto al género destaca notablemente el femenino $(80,7 \%)$ frente al masculino $(19,3 \%)$. Un $29,5 \%$ de los encuestados cursa estudios en el Grado de Educación Infantil, el $14.8 \%$ de los encuestado estudia el Grado de Educación Primaria y el 55,7\% cursa el Máster de Formación del Profesorado. De los encuestados que cursa los estudios de Grado el $31,8 \%$ lo hace en primero y el $68,2 \%$ lo hace en tercero. En cuanto a la edad de los participantes, un $75 \%$ oscila entre los 19 años y los 16 años. El 15,3\% indica que cuenta con 3 Apps en su dispositivo móvil, el 60,2\% tiene entre 5 y 20 , y el $24 \%$ indica que tiene más de 20, y el 0,5\% dice no tener descargada ninguna Apps. Finalmente, el 65,2\% indica que sí ha utilizado Apps para aprender idiomas.

\section{Instrumento}

Para la recogida de datos tuvimos presente que los diseños de investigación descriptiva despuntan entre los métodos o diseños para la investigación cuantitativa considerándose procedimientos en los que el investigador administra un instrumento a una muestra o población de sujetos de estudio para describir actitudes, creencias, opiniones... (Creswell, 2002).

Revisados los instrumentos disponibles y las limitaciones para este estudio decidimos elaborar cuestionario on-line anónimo e individual diseñado ad hoc, con el fin de medir el uso que hacen los alumnos universitarios del Grado de Maestro y Máster de Formación del Profesorado de la Universidad de Murcia de Apps para el aprendizaje de idiomas. Para el diseño del cuestionario tuvimos presente los siguientes requisitos: brevedad, sencillez, vocabulario comprensible y adaptado a las características de la muestra, motivador, atractivo en su diseño y soporte virtual que facilite su cumplimentación (Ramos, Giménez, Lapaz y Muñoz, 2006).

El cuestionario está compuesto por una serie de ítems que hacen referencia al uso que hacen de las Apps, en qué medida las utilizan para el aprendizaje de idiomas y qué habilidades desarrolla con el uso de las Apps. Consta de dos módulos: el primero recoge datos sociodemográficos: género, edad, estudios, utilización de dispositivos móviles, y de las preguntas "¿Cuántas Apps tienes en tu dispositivo móvil (smartphone, tableta, ...)?" y "para aprender un idioma ¿utilizas las Apps?". El segundo modulo está compuesto por 29 ítems agrupados en siete dimensiones: habilidades en el dominio de dispositivos móviles, conocimiento de las Apps, frecuencia en qué te descargas, grado

Apps para el aprendizaje de idiomas en la Universidad de Murcia. $\mathrm{M}^{\mathrm{a}}$ Encarnación CarrilloGarcía, Antonia Cascales-Martínez, y Amando López Valero. 
de conocimiento de las Apps, para que utilizas las Apps en el aprendizaje de idiomas, el uso de las Apps en el ámbito universitario y, finalmente, en qué medida las Apps ayudan al aprendizaje de idiomas para toda la vida. Las preguntas son de respuesta cerrada tipo Likert, a las que se responde según una escala de menor a mayor grado de acuerdo: de 1 totalmente en desacuerdo a 5 totalmente de acuerdo.

La propia naturaleza del instrumento que parte de las inquietudes de los autores tras consultar la bibliografía estudiada aconsejaba su validación de contenido con una estrategia de fiabilidad entre jueces de carácter cualitativo, para lo cual se contó con cinco expertos: una doctora en pedagogía y profesora de universidad, dos profesoras titulares de universidad y expertas en tecnologías educativa, dos ingenieros informáticos. Se solicitó a los expertos que valorasen diferentes aspectos sobre los datos iniciales sociodemográficos, las dimensiones, los ítems, opciones de respuesta, y una valoración global (Wieserma, 2001). Tras dicha validación se realizaron mínimas aportaciones al cuestionario como fueron la incorporación de una respuesta a una de las preguntas cerradas y algunas modificaciones de redacción.

Realizado el análisis de la fiabilidad del instrumento, hemos obtenido una alta consistencia interna, arrojando un coeficiente de Alfa de Cronbach de 0,959 siendo, según De Vellis (2003), excelente al obtener una puntuación mayor de 0,90.

\section{Resultados}

A continuación, exponemos los resultados de los análisis procedentes de la información recogida con el cuestionario de evaluación cumplimentado por los alumnos.

Objetivo 1. Analizar la percepción que tienen los alumnos sobre las Apps para el aprendizaje de los idiomas.

En la Tabla I se muestran los estadísticos descriptivos para siete dimensiones del cuestionario que determina la percepción de los alumnos sobre el uso de las Apps para el aprendizaje de los idiomas. La puntuación más alta se revela en la dimensión dominio de habilidades de dispositivos móviles $(X=4.14)$, y el valor medio más bajo se ha obtenido en la dimensión Apps como ayuda en el aprendizaje de idiomas a lo largo de toda la vida $(X=1,88)$.

Tabla I.

Estadísticosdel cuestionario sobre las Apps como herramientas para el aprendizaje de idiomas.

\begin{tabular}{llllllll}
\hline Dimensiones & & Min. & Max & Moda & Mediana & Media & $\begin{array}{l}\text { Desv. } \\
\text { Típica }\end{array}$ \\
\hline $\begin{array}{l}\text { A. Dominio de } \\
\text { habilidades }\end{array}$ & $\begin{array}{l}\text { de } \\
\text { dispositivos móviles. }\end{array}$ & & 5 & 4 & 4,00 & 4,14 &, 746 \\
$\begin{array}{l}\text { B. Grado de } \\
\text { conocimiento de las }\end{array}$ & & 5 & 4 & 4,00 & 3,61 &, 823 \\
\end{tabular}

Apps para el aprendizaje de idiomas en la Universidad de Murcia. $\mathrm{M}^{\mathrm{a}}$ Encarnación CarrilloGarcía, Antonia Cascales-Martínez, y Amando López Valero. 


\begin{tabular}{|c|c|c|c|c|c|c|}
\hline $\begin{array}{l}\text { C. Frecuencia de } \\
\text { descarga de Apps. }\end{array}$ & 1 & 5 & 2 & 2,00 & 2,45 & ,856 \\
\hline $\begin{array}{l}\text { D. Valoración de las } \\
\text { características de las } \\
\text { Apps. }\end{array}$ & 1 & 5 & 4 & 4,00 & 3,99 & 899 \\
\hline $\begin{array}{lr}\text { E. Habilidades } \\
\text { vinculadas } \\
\text { aprendizaje de un } \\
\text { idioma que se } \\
\text { desarrollan con las } \\
\text { Apps. }\end{array}$ & 1 & 5 & 3 & 3,00 & 2,98 & 1,058 \\
\hline $\begin{array}{l}\text { F. Apps en el ámbito } \\
\text { universitario. }\end{array}$ & 1 & 5 & 3 & 3,00 & 2,80 & 1,136 \\
\hline $\begin{array}{l}\text { G. Apps como ayuda } \\
\text { en el aprendizaje de } \\
\text { idiomas a lo largo de } \\
\text { toda la vida. }\end{array}$ & 1 & 4 & 1 & 1,00 & 1,88 & 1,091 \\
\hline
\end{tabular}

$\mathrm{Al}$ analizar los resultados, el $84.1 \%$ de los encuestados dice tener un dominio bueno o excelente del manejo de dispositivos móviles, frente a $1 \%$ que no domina los dispositivos móviles. Respecto al conocimiento de las Apps, el 39,8\% considera que las conoce, el 40,9\% las conoce bien y el 13,6\% el grado de conocimiento es muy alto. En la frecuencia de descarga hay mayor disparidad de respuesta, un 19,3\% considera que apenas se descarga Apps, 36,9\% se descarga pocas Apps, frente a un 38,6\% que indica reconoce que se descarga bastantes y muchas.

El 76,2\% de los participantes consideran que las Apps son fáciles o muy fáciles de usar. El 92\% de los encuestados ha respondido que las Apps son muy cómodas dado que se puede acceder a ellas en cualquier momento. El 63,7\% indican que estas aplicaciones les entretienen, frente a $6,8 \%$ que opinan lo contrario. Los encuestados opinan que las Apps son útiles o muy útiles $72,7 \%$, mientras que un $1,1 \%$ que indican que no son nada útiles. El 37,5\% de los alumnos encuestados considera que las Apps les permiten interactuar mucho con otros usuarios y el 35,2\% le permiten interactuar con otros usuarios, frente al $9.1 \%$ que consideran que se interactúa poco. Los $89,3 \%$ encuestados consideran que se puede aprender con las Apps. Asimismo, 87,5\% de los alumnos participantes han considerado que las Apps son un recurso educativo, si bien hay variaciones en el grado. Al preguntar por las Apps como recurso educativo en el ámbito universitario, nos encontramos que el $13,6 \%$ de los encuestados consideran que no lo son, el $14,8 \%$ lo consideran un poco, frente al $20,5 \%$ que consideran que son verdaderos recursos educativos. Cuando preguntamos sobre las Apps como recurso educativo para la enseñanza de idiomas, los encuestados opinan que un $29,5 \%$ consideran que nada o

Apps para el aprendizaje de idiomas en la Universidad de Murcia. M $^{\mathrm{a}}$ Encarnación CarrilloGarcía, Antonia Cascales-Martínez, y Amando López Valero. 
muy poco, mientras que $70,5 \%$ de los alumnos encuestados entienden que sí en mayor o menor medida.

El $72,7 \%$ de los encuestados creen que las Apps son recursos para la enseñanza de idiomas en el ámbito universitario, mientras que $84,1 \%$ las consideran como recursos para el aprendizaje de idiomas en el ámbito universitario; un porcentaje muy similar, el $78,4 \%$ las consideran como recursos que favorecen el auto-aprendizaje de idiomas.

Les preguntamos a los alumnos universitarios en qué medida mejoran las habilidades específicas para el aprendizaje de idiomas. Un 39,8\% de los encuestados consideran que las Apps no mejoran la comprensión oral, frente a un $27,3 \%$ que sí, un $33 \%$ no se define. Respecto a la expresión oral, un 33\% consideran que las Apps no mejoran esta habilidad; sin embargo, un 40,5\% consideran que sí, y un 39,8\% no lo tienen muy claro. El 34,1\% de los encuestados entienden que las Apps mejora la comprensión, y un 33\% entienden que no lo hace. En lo que se refiere a la expresión escrita, el 31,8\% consideran que las Apps no la mejoran, y el 28,4\% consideran que estas aplicaciones móviles sí mejoran esta habilidad. Respecto a la fonética, el 46,6\% entienden que se ve favorecido, mientras que el 20,5\% no lo hace. Las Apps como recurso de evaluación, el $63,7 \%$ de los alumnos encuestados entienden que sí lo son frente al 15,9 que no lo son.

Preguntamos explícitamente sobre el uso de las Apps en las aulas universitarias, y solo un $25 \%$ indica que se utilizan para impartir docencia. El 45,5\% de los encuestados manifiestan que son un complemento de la enseñanza. En esa misma línea, el 70,4 \% indica que las Apps que facilitan el aprendizaje de idiomas no se comparten entre compañeros.

Finalmente, les preguntamos en qué medida las Apps ayudan en el aprendizaje de idiomas a lo largo de toda la vida. El 69,3\% de los estudiantes indicaron que no mientras que solo un $11,4 \%$ contestaron que sí.

Objetivo 2. Analizar las posibles diferencias entre la percepción que tienen los alumnos sobre las Apps para el aprendizaje de los idiomas en relación al género y a la titulación.

A fin de determinar las relaciones existentes entre las diferentes variables implicadas, un objetivo característico de esta modalidad de investigación, las variables predictoras consideradas han sido: género, edad, grado que estudias, Apps que dispones en tu dispositivo móvil, y en qué medida utilizas las Apps para aprender un idioma. Por su parte, las variables criterio que se han estimado son: habilidad con los dispositivos móviles, grado de conocimiento de Apps, frecuencia de descargas de Apps, características de las Apps, las Apps en la enseñanza de idiomas, las Apps en las aulas universitarias y en qué medida las Apps ayudan en el aprendizaje de idiomas para toda la vida. Para ello la hipótesis nula (H0) hace referencia a la no existencia de diferencias significativas asumiendo un valor de probabilidad de error de $\alpha=.05$ o inferior, y la alternativa (H1) a la existencia de tales diferencias en las mismas condiciones

Al cruzar los datos de la categoría de titulación, entre los alumnos que cursan el Máster de Formación del Profesorado y el Grado de Maestro con las variables predictoras no encontramos diferencias estadísticamente significativas según los datos arrojados tras el cálculo de la prueba de $U$ de Mann Whitney. Al realizar ese mismo cruce en función del género, encontramos diferencias estadísticamente significativas en las habilidades que se desarrollan en el aprendizaje de idiomas: comprensión oral y escrita, y expresión oral

Apps para el aprendizaje de idiomas en la Universidad de Murcia. $\mathrm{M}^{\mathrm{a}}$ Encarnación CarrilloGarcía, Antonia Cascales-Martínez, y Amando López Valero.

Página 11 de 18 
y escrita $(p<0.05)$ siendo las chicas las que opinan que con las Apps se aprende un idioma.

Objetivo 3. Determinar la relación existente entre las diferentes habilidades (comprensión oral y escrita y expresión oral y escrita) para aprender un idioma con Apps.

Finalmente hemos realizado la comparación entre las variables propias del aprendizaje de idiomas, como son la comprensión oral y escrita, y la expresión oral y escrita para determinar el nivel de significación. Al analizar la relación entre los resultados obtenidos en el cuestionario en cada uno de los ítems valor en la prueba $T$ de Wilcoxon es $p<0.05$; por lo tanto se puede concluir que existe una relación estadísticamente significativa entre las cuatro habilidades. Si nos detenemos en el detalle, según la percepción de los alumnos, cuando comparamos la comprensión oral y la comprensión escrita ( $R P$ comprensión oral $22,63>R P$ comprensión escrita 20,94$)$ los datos revelan que, según los encuestados, las Apps son un recurso más apropiado para desarrollar la comprensión oral. Esta relación también se establece con la expresión oral ( $R P$ comprensión oral 24,69) $R P$ expresión oral 16,67$)$ y la expresión escrita oral $(R P$ comprensión oral $23,74>R P$ expresión escrita 17,50). Al comparar la comprensión escrita la expresión oral y escrita los datos revela una relación estadísticamente significativa desfavorable en ambos casos a la expresión oral ( $R P$ comprensión escrita 16,27> $R P$ expresión oral 1,00; $R P$ expresión escrita 20,85> $R P$ expresión oral 16,18$)$. Los alumnos perciben que la expresión oral no es la habilidad que mejor se aprende con las Apps. Finalmente, comparamos la expresión oral y escrita y los resultados no demuestran que existe una relación estadísticamente significativa $(p>$ $0.05)$.

\section{Discusión}

De los resultados de este estudio se derivan determinadas inferencias que ponen en entredicho el uso de la tecnología en las aulas universitarias, a pesar de que las tecnologías han cambiado nuestra forma no sólo de comunicarnos y relacionarnos sino también de aprender, y en especial una lengua extranjera. Este resultado es coincidente con otras investigaciones realizadas (Blake, 2015; Cascales, Gomariz y MartínezSegura, 2016; Gunter, Campbell, Braga, Racilan y Souza, 2016; Marcelo, Yot y Mayor, 2015).

A través del marco teórico descrito en esta investigación se ha puesto de manifiesto la influencia de las tecnologías, y en especial del mobile learning, en el ámbito educativo, lo que conlleva un mayor uso de las aplicaciones para este tipo de dispositivos, también llamadas Apps . Si bien, el resultado de nuestro estudio concluye que la Apps, según los alumnos, son herramientas adecuadas para el aprendizaje de los idiomas por otro lado no se utilizan como tales en los diferentes entornos educativos, universitarios y no universitarios. De igual modo, los alumnos consideran que la utilización de este tipo de recursos hace que se amplíen e incrementen las posibilidades de aprendizaje de idiomas (Blake, 2015; Jones, Kna, Jukulska-Hulme y Eardley, 2016; Rico y Agudo, 2016). Aunque los estudiantes reconocen que no comparten con sus compañeros las aplicaciones y recursos que utilizan para el aprendizaje de idiomas.

Según la investigación realizada por Henze et al. (2011) las Apps han alcanzado un auge impresionante, pero su utilización está restringida a periodos cortos de tiempo,

Apps para el aprendizaje de idiomas en la Universidad de Murcia. M $^{\mathrm{a}}$ Encarnación CarrilloGarcía, Antonia Cascales-Martínez, y Amando López Valero.

Página 12 de 18 
coincidiendo la valoración realizada por los alumnos participantes. Consideran que las Apps no ayudan en el aprendizaje de idiomas a lo largo de toda la vida. Este hecho contrasta con la relevancia que se le está dando al uso la tecnología en los contextos educativos (Hernández, 2015; Lozano, 2011; Ozuorcun y Tabak, 2012).

Ahora bien, al revisar los resultados en función de las habilidades propias del aprendizaje de idiomas, aparece una relación estadísticamente significativa entre las habilidades de comprensión y expresión oral y comprensión escrita, indicando que los alumnos entienden que aprenden mejor con las Apps. El resultado es similar al hallado en las investigaciones realizadas por Alvarado et al. (2016), Demouy et al. (2016), García et al. (2016), Gunter, et al (2016), Lindaman y Nolan (2015) y Martínez et. al (2016), que también consideraron que las Apps ayudan a trabajar de manera práctica los contenidos de la lengua e incrementan exponencialmente las oportunidades de aprendizaje. Por lo que el desarrollo de la comprensión escrita queda relegada a otro tipo de recursos tecnológicos o métodos de enseñanza, según las opiniones de los participantes en este estudio.

A la luz de los datos obtenidos y analizados queremos poner de manifiesto que consideramos las Apps recursos muy adecuados para favorecer el aprendizaje de los idiomas, en el especial dentro de la formación de los futuros docentes dado que puede favorecer el desarrollo de estrategias pedagógicas de cara a la planificación e implementación de la enseñanza. De esta manera, el docente interesado en utilizar este recurso educativo debe ponerlo al servicio de la consecución de los objetivos educativos de su programación docente, y así a través de ellas trabajar los contenidos planificados, en este caso los contenidos relacionados con la enseñanza de un segundo idioma. Todo ello se verá favorecido si se hace una adecuada selección de actividades, por medio de las diferentes aplicaciones móviles que se seleccionen, que como característica principal deben favorecer la práctica de los diferentes contenidos del idioma y por consecuencia ser favorecedoras de la consecución de los objetivos de enseñanza y aprendizaje. También destacar el papel que la evaluación debe tener en todo este proceso, pues sin una adecuada evaluación no se consigue una óptima implementación. Por tanto, y en cuanto a la evaluación se refiere, las Apps deben ayudar en los diferentes momentos de la evaluación del proceso educativo, esto es, se han de buscar que permitan o ayuden con la evaluación inicial, procesual y final. También y con el objetivo de ir mejorando los procesos de implementación con estos recursos móviles se debe hacer una evaluación del proceso educativo, el papel de la labor docente con respecto a los mismos, así como evaluar las diferentes Apps utilizadas en el proceso. En relación a este aspecto, se puede decir que la evaluación de las Apps, con respecto a su papel en el proceso educativo, proporcionará los datos adecuados para determinar si la selección de dicha aplicación ha favorecido la consecución de los objetivos, permitiendo a partir de la misma tomar las convenientes decisiones a la hora de la selección de las mejores Apps para el aprendizaje de idiomas.

Para finalizar, queremos indicar que este trabajo no está libre de limitaciones, dado que nos hemos centrado en un perfil concreto de alumnos, curso y titulación, lo que ha condicionado claramente la muestra. Sería interesante, ampliar este estudio a otros cursos y titulaciones, hecho que lo plantearemos para un trabajo futuro.

Consideramos que nuestro objetivo ha sido cubierto y el resultado obtenido puede contribuir a una mejor comprensión sobre aquellos factores que influyen en el aprendizaje de los idiomas dentro del Mobile Learning, en especial con las Apps. De

Apps para el aprendizaje de idiomas en la Universidad de Murcia. $\mathrm{M}^{\mathrm{a}}$ Encarnación CarrilloGarcía, Antonia Cascales-Martínez, y Amando López Valero.

Página 13 de 18 
esta forma, puede aportar una nueva visión sobre cómo abordar eficazmente en las universidades el reto del uso de las Apps en el aprendizaje de los idiomas, integrando como medios facilitadores las tecnologías emergentes. El objetivo de esta línea de investigación es seguir ampliando el campo de estudio, con el fin de compartir con la comunidad científica los diferentes análisis que de ello deriven.

Fecha de redacción: 23 de marzo de 2017

Presentación del artículo: 5 de junio de 2018 Fecha de aprobación: 11 de septiembre de 2018

Fecha de publicación: 31 de octubre de 2018

Carrillo-García, M.E., Cascales-Martínez, A., y López Valero, A. (2018). Apps para el aprendizaje de idiomas en la Universidad de. RED. Revista de Educación a Distancia, 58. Consultado el (dd/mm/aaaa) en http://www.um.es/ead/red/58/

\section{Financiación}

Esta investigación no ha recibido ninguna subvención específica de los organismos de financiación en los sectores públicos, comerciales o sin fines de lucro.

\section{Referencias}

Alvarado, N. C., Coelho, D., y Dougherty, E. (2016). "Mobile apps for ELLs: Supporting language learning with engaging digital tools". AJAL, 43. Consultado el $10 / 01 / 17$ http://www.faapi.org.ar/ajal/issues/401/AJALVol4(1).pdf\#page=44.

Área Moreira, M. (2010). Competencias informacionales y digitales en educación superior. RuscVol. 7, $\mathrm{n}^{\mathrm{o}}$ 2, Universidad Oberta de Catalunya. Barcelona, julio de 2010. Consultado el 18/01/17 en http://hdl.handle.net/10609/2601.

Área Moreira, M., Hernández Rivero, V. y Sosa Alonso, J. J. (2016). Modelos de integración didáctica de las TIC en el aula. Comunicar, $\mathrm{n}^{\circ}$ 47, v. XXIV, Revista Científica de Educomunicación. 79-87. Consultado el 25/01/17 en http://dx.doi.org/10.3916/C47-2016-08.

Avello, Raidell; López, Raúl; Vázquez, Silvia. (2016). Competencias TIC de los docentes de las escuelas de Hotelería y Turismo cubanas. Universidad y Sociedad, vol. 8, núm. 1, pp. 63-69.

Blake, R. (2015). Technology and the Four Skills. En Godwin-Jones, R. (2016). Emerging technologies looking back and ahead: 20 years of technologies for language learning. About Language Learning \& Technology, 5. Consultado el 10/01/17 en http://llt.msu.edu/issues/june2016/blake.pdf.

Brown, D. (1994). Principles of Language Learning and Teaching, Prentice Hall Regents.

Apps para el aprendizaje de idiomas en la Universidad de Murcia. $\mathrm{M}^{\mathrm{a}}$ Encarnación CarrilloGarcía, Antonia Cascales-Martínez, y Amando López Valero. 
Brumfit, C.J. \& Johnson, K. (1991). The Communicative Approach to Language Teaching, Oxford, Oxford University Press.

BURBAT, R. (2016). El aprendizaje autónomo y las TIC en la enseñanza de una lengua extranjera: ¿Progreso o retroceso?. PORTA LINGUARUM, 37-51. Consultado el $30 / 03 / 2019$ en

Cabero Almenara, J., Arancibia Muñoz, M. L., Valdivia Zamorano, I., \&Aranedas Riveros, S. M. (2018). Percepciones de profesores y estudiantes de la formación virtual y de las herramientas en ellas utilizadas. Revista Diálogo Educacional, 18(56), 149-163. https://idus.us.es/xmlui/handle/11441/71505

Cascales Martínez, A., Martínez Segura, M. J., yGomariz Vicente, M. Á. (2016). Competencia tecnológica y trabajo colaborativo en las prácticas curriculares del Grado en Pedagogía en la Universidad de Murcia. Revista de Investigación en Educación, 14(1), 31-52. Consultado el 16/02/17 en http://reined.webs.uvigo.es/ojs/index.php/reined/article/view/1135/371\#page=36.

Correa, J., Fernández, L., Gutiérrez, A., Losada, D., Ochoa-Aizpurua, B. (2015). Formación del profesorado, tecnología educativa e identidad docente digital. RELATEC, vol. 14, núm. 1, pp. 45-56. http://dx.doi.org/10.17398/1695288X.14.1.45

Creswell, J. (2002). Educational Research: Planning, conducting and evaluating. Quantitative and qualitative research. Ohio: Merrill Prentice Hall.

De Vellis, R.F. (2003). Scale development: Theory and applications (2 $2^{\mathrm{a}}$ ed.). Thousand Oaks, CA: Sage.

Del Moral, Maríar; Villalustre, Lourdes; Neira, María. (2013). Oportunidades de las TIC para la innovación educativa en las escuelas rurales de Asturias. Aula Abierta, vol. 42, núm. 1, pp. 61-67. http://dx.doi.org/10.1016/S02102773(14)70010-1

Demouy, V., Jones, A., Kan, Q., Kukulska-Hulme, A., \& Eardley, A. (2016). Why and how do distance learners use mobile devices for language learning?.The Euro CALL Review, 24(1), 10-24. Consultado el 13/03/17 en http://dx.doi.org/10.4995/eurocall.2016.5663.

Durán-Fernández, A., \& Barrio-Barrio, J.F. (2007). Disposición y uso de recursos informáticos para la enseñanza-aprendizaje del inglés: una descripción a partir de una muestra en cien centros públicos de educación infantil y primaria de la Comunidad de Madrid. Porta Linguarum, 8, 193-223. (http://goo.gl/ehtyrd) (2016-04-30).

Fajardo, I., Villalta, E. y Salmerón, L. (2016): ¿Son realmente tan buenos los nativos digitales? Relación entre las habilidades digitales y la lectura digital. En Anales de Psicología, 2016, vol. 32, $\mathrm{n}^{\circ} 1$ (enero, 89-97). Consultado el 10/02/17 en http://dx.doi.org/10.6018/analesps.32.1.185571

Felix, u. (2004). a Multivariate analysis of secondary students' experience of Webbased language learning. ReCALL, 16, 129-141. https://doi.org/10.1017/s0958344004001715

Apps para el aprendizaje de idiomas en la Universidad de Murcia. $\mathrm{M}^{\mathrm{a}}$ Encarnación CarrilloGarcía, Antonia Cascales-Martínez, y Amando López Valero. 
García Corcés, M., Mosquera Gende, I., y Marro Roig, B. C. (2016). Recursos innovadores para la adquisición de destrezas orales en la lengua inglesa en la etapa de Educación Infantil. I Congreso Internacional de Innovación y Tecnología Educativa en Educación Infantil (2016), p 1-11 (pp. 1-11). Consultado el 10/02/17 en https://idus.us.es/xmlui/handle/11441/43634 .

González, A. J. G., Gómez-Millán, M. R. B., \& Rubio, L. R. (2017). Competencias comunicativas mediadas en estudiantes universitarios mayores. Alfabetización tecnológica como experiencia innovadora. RELATEC: Revista Latinoamericana de Tecnología Educativa, 16(1), 67-77. Consultado el 30/09/18 en https://dialnet.unirioja.es/servlet/articulo?codigo $=6046928$

Gunter, G. A., Campbell, L. O., Braga, J., Racilan, M., \& Souza, V. V. S. (2016). Language learning apps or games: an investigation utilizing the RETAIN model. Revista Brasileira de Linguística Aplicada, (AHEAD), 0-0. Consultado el 03/02/17 en http://dx.doi.org/10.1590/1984-639820168543.

Henze, N., Pielot, M., Poppinga, B., Schinke, T. \& Boll, S. (2011).“My Apps is an experiment: Experience from User Studies in Mobile Apps Stores". International Journal of Mobile Human Computer Interaction, v. 3, n.4, pp. 71-91. Consultado el 28/02/17 en http://dc.doi.org/10.4018/jmhci.2011100105

Hernández Salazar, P. (2015). Experiencias de Alfabetización Informativa en Ambientes Virtuales de Aprendizaje. Biblios. Revista de Biblioteconomía y Ciencias de la Información, $n^{o}$ 61. 19-37. Consultado el 03/01/17 en http://dx.doi.org/10.5195/biblios.2015.254.

Izquierdo, J., Cruz, V. D. L., Aquino Zúñiga, S. P., Sandoval Caraveo, M. C., \& García Martínez, V. (2017). La enseñanza de lenguas extranjeras y el empleo de las TIC en las escuelas secundarias públicas.

Izquierdo, N. C. (2008). La enseñanza-aprendizaje de lenguas extranjeras y las TICs: el caso del Español como Lengua Extranjera (ELE). Iniciación a la Investigación, $(3 \mathrm{e})$.

Lázaro-Cantabrana, J. L., Gisbert-Cervera, M., \& Silva-Quiroz, J. E. (2018). Una rúbrica para evaluar la competencia digital del profesor universitario en el contexto latinoamericano. Edutec. Revista Electrónica de Tecnología Educativa, (63), 1-14. Cosultado en http://www.edutec.es/revista/index.php/edutece/article/view/1091/pdf

Lindaman, D., \& Nolan, D. (2016). Mobile-Assisted Language Learning: Application Development Projects Within Reach for Language Teachers. IALLT Journal of Language Learning Technologies, 45(1), 1-22. Consultado el 21/02/17 en https://goo.gl/v7JblJ.

López, L. y Aguadez, M. C. (2015). La docencia sobre alfabetización mediática en las facultades de Educación y Comunicación. Comunicar, n 44, v. XXII. Revista Ciéntífica de Educomunicación. 187-195. Consultado el 01/02/17 en http://dx.doi.org/10.3916/C44-2015-20

López, M. A. R., Sánchez, V. M. G., \& Padilla-Hernández, A. L. (2018). Selección de categorías para el estudio de la evolución de la competencia digital docente del profesorado en Educación Superior. Revista Interuniversitaria de Investigación en

Apps para el aprendizaje de idiomas en la Universidad de Murcia. $\mathrm{M}^{\mathrm{a}}$ Encarnación CarrilloGarcía, Antonia Cascales-Martínez, y Amando López Valero.

Página 16 de 18 
Tecnología Educativa, (4), 55-67. Consultado en http://revistas.um.es/riite/article/view/327881/231811

Macaro, e., handley, Z., \& Walter, C. (2011). a systematic review of Call in english as a second language: Focus on primary and secondary education. (state-of-the-art article). Language Teaching, 45(1), 1-43. https://doi.org/10.1017/s0261444811000395

Marcelo, C., Yot, C. y Mayor, C. (2015). Enseñar con tecnologías digitales en la Universidad. Comunicar, $\mathrm{n}^{\mathrm{o}}$ 45, V. XXIII. Revista Científica de Educomunicación. 118-124. Consultado el 01/03/17 en http://dx.doi.org/10.3916/C45-2015-12

Martínez Parejo, R. (2016). Desarrollo de la competencia escrita en la enseñanza de lenguas extranjeras a través del uso de dispositivos móviles. Revista Complutense de Educación, Vol 27, $\mathrm{n}^{\mathrm{o}}$ 2. 779-803. Consultado el 16/02/17 en https://goo.gl/gSCnl5

Monroy García, F. A., Masa, H., \& Francisco, J. (2018). Conocimiento y utilización general de las TIC que presentan los estudiantes universitarios. In Avances en democracia y liderazgo distribuido en educación: Actas del II Congreso internacional de liderazgo y mejora de la educación. Red de Investigación sobre Liderazgo y Mejora de la Educación (RILME). Consultado en https://repositorio.uam.es/handle/10486/683108

Moreno Martínez, N. M., Leiva Olivencia, J. J. y Matas Terrón, A. (2016). Mobile learning, Gamificación y Realidad Aumentada para la enseñanza-aprendizaje de idiomas. IJERI: International Journal of Educational Research and Innovation, (6), 16-34. Consultado el 11/02/17 en https://goo.gl/tqb4lb

Ojeda, G. S., Reyes, G. G. E., \& Reyes, H. D. R. (2018). Programa de alfabetización digital para formadores de docentes. RECIE. Revista Electrónica Científica de Investigación Educativa,4(1), 691-708. Consultado el 30/09/18 en http://www.rediech.org/ojs/2017/index.php/recie/article/view/340

Ozuorcun, N. C., \&Tabak, F. (2012). Is M-learning Versus E-learning or are They Supporting Each Other? Procedia-Social and Behavioral Sciences, 46, 299-305. Consultado el 10/01/17 en http://dx.doi.org/10.1016/j.sbspro.2012.05.110 .

Piñero Otero, T. y Costa Sánchez, C. (2015). ARG (juegos de realidad alternativa). Contribuciones, limitaciones y potencialidades para la docencia universitaria. Comunicar, n 44, v. XXII. Revista Científica de Educomunicación. 141-148. Consultado el 02/02/17 en http://dx.doi.org/10.3916/C44-2015-15.

Porlán, I. G., Espinosa, M. P. P., \& Sánchez, F. M. (2018). Competencia digital: una necesidad del profesorado universitario en el siglo XXI. RED: Revista de Educación a Distancia, (56), 7. Consultado en https://dialnet.unirioja.es/servlet/articulo? codigo $=6501069$

Rico García, M. M. y Agudo Garzón, J. E. (2016). Aprendizaje móvil de inglés mediante juegos de espías en Educación Secundaria. Revista Iberoamericana de Educación a Distancia, 19 (1), 121. Consultado el 14/01/17 en https://goo.gl/292nzF.

Apps para el aprendizaje de idiomas en la Universidad de Murcia. $\mathrm{M}^{\mathrm{a}}$ Encarnación CarrilloGarcía, Antonia Cascales-Martínez, y Amando López Valero. 
Rodríguez, M. D. M., Méndez, V. G., \& Martín, A. M. R. M. R. (2018). ALFABETIZACIÓN INFORMACIONAL Y COMPETENCIA DIGITAL EN ESTUDIANTES DE MAGISTERIO. Profesorado, Revista de Currículum y Formación del Profesorado,22(3), 253-270. Consultado el 30/09/18 en http://revistaseug.ugr.es/index.php/profesorado/article/view/8001

Sánchez Prieto, J. C., Olmos Migueláñez, S., García-Peñalvo, F. J. (2017). ¿Utilizarán los futuros docentes las tecnologías móviles? Validación de una propuesta de modelo TAM extendido. Red. Revista de Educación a Distancia. 52, 5. Consultado el 01/01/18 en https://goo.gl/CmU8T6

Tejada Fernández, J., \&Pozos Pérez, K. V. (2018). Nuevos escenarios y competencias digitales docentes: hacia la profesionalización docente con TIC. Profesorado, Revista de Currículum y Formación del Profesorado, 22(1), 41-67. Consultado en https://recyt.fecyt.es/index.php/profesorado/article/view/63620

Wieserma, L.D. (2001). Conceptualization and development of the sources of enjoyment in youth sport questionnaire. Measurement in Physical Education and Exercise Science, 5 (3), 153-157. Consultado el 03/03/17 en http://dx.doi.org/10.1207/S15327841MPEE0503_3

Zempoalteca Durán, B., Barragán López, J., González Martínez, J. \& Guzmán Flores, T. (2017). Formación en TIC y competencia digital en la docencia en instituciones públicas de educación superior. Apertura (Guadalajara, Jal.), 9(1), 80-96. https://dx.doi.org/10.18381/Ap.v9n1.922

Apps para el aprendizaje de idiomas en la Universidad de Murcia. $\mathrm{M}^{\mathrm{a}}$ Encarnación CarrilloGarcía, Antonia Cascales-Martínez, y Amando López Valero. 